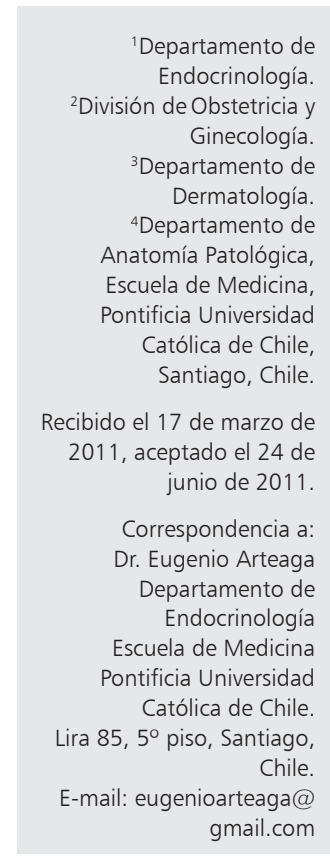

\section{Hiperandrogenismo avanzado en una mujer postmenopáusica. Caso clínico}

\author{
PATRICIO SALMAN M. ${ }^{1}$, MAURICIO CUELLO F. ${ }^{2}$, MARIANNE KOLBACH ${ }^{3}$, \\ ROGER GEJMAN ${ }^{4}$, EUGENIO ARTEAGA U. ${ }^{1}$

\section{Virilization caused by an ovarian tumor. Report of one case}

We report a 76-year-old woman with a virilization syndrome characterized by progressive androgenic alopecia, clitoris enlargement and hirsutism predominating in the face. Plasma testosterone was $711 \mathrm{ng} / \mathrm{dl}$. Magnetic resonance imaging showed slightly enlarged ovaries with a cyst in the left. A bilateral oophorectomy was performed, demonstrating the presence of a Leydig cell hilar tumor in the right ovary. The patient had a good postoperative evolution with reduction of androgen levels and reversion of alopecia. (Rev Med Chile 2011; 139: 1066-1070).

Key words: Hyperandrogenism; Leydig cell tumor; Testosterone.
L

os casos de virilización en la postmenopausia son extraordinariamente raros y suelen deberse a causas tumorales. La orientación diagnóstica depende de la severidad del cuadro y rapidez de evolución ${ }^{1}$. El diagnóstico etiológico puede ser difícil debido a que el tumor, especialmente el ovárico, no es fácilmente demostrable por la imagenología. El objeto del presente trabajo es presentar el caso excepcional de una mujer postmenopáusica con virilización de evolución prolongada que correspondió a un tumor ovárico de Leydig hilar y que revirtió con la ooforectomía bilateral.

\section{Caso clínico}

Mujer de 76 años con alopecía de tipo androgénica de predominio frontal, grado IV de la escala de Ludwig, de dos años de evolución, asociado a hirsutismo facial, conducta más agresiva y exacerbación de su libido. Sin historia compatible con hipercortisolismo. Menopausia a los 54 años y ausencia de flujos rojos posteriores. Sin antecedente de uso de terapia hormonal de reemplazo. La evaluación endocrinología confirmó el hiperandrogenismo (Tabla 2).
Entre sus antecedentes destacaba hipertensión arterial controlada con de amlodipino 5 $\mathrm{mg}$ /día e hidroclorotiazida $25 \mathrm{mg}$ /día asociada a triamtereno $50 \mathrm{mg} /$ día, dislipidemia mixta en tratamiento con dieta y bezafibrato $400 \mathrm{mg} /$ día, gota en tratamiento con alopurinol y colchicina, y uso profiláctico de omeprazol $20 \mathrm{mg} /$ día por antecedente de úlcera gástrica.

De su examen físico destacaba la alopecía androgénica (Figura 1), score Ferriman-Gallway de $7(\mathrm{VN}<6)$, trofismo muscular aumentado, piel untuosa, leve clitoromegalia $(1,5 \times 1 \mathrm{~cm})$ y mucosa vaginal estrogenizada y lubricada.

El estudio bioquímico inicial (Tablas 1 y 2 ) mostraba poliglobulia (la hemoglobina subió de $12,5 \mathrm{~g} / \mathrm{dL}$ en el año 2004 a 17,8 al momento del estudio en el 2009), testosterona plasmática de 711 ng/dL, DHEA-S indetectable, elevación moderada de estradiol $(48 \mathrm{pg} / \mathrm{ml})$ y gonadotropinas insuficientemente elevadas (FSH 16,9 mUI/ml y LH 9,3 $\mathrm{mUI} / \mathrm{ml}$ ) para su condición de postmenopáusica. El cortisol libre urinario fue normal.

La resonancia magnética (RM) de abdomen y pelvis demostró esteatosis hepática difusa, suprarrenales de aspecto normal, adenomiosis uterina, ovario derecho de $20 \times 10 \mathrm{~mm}$ y ovario izquierdo de $35 \times 20 \mathrm{~mm}$ con pequeño quiste simple de 17 
Tabla 1. Estudio bioquímico general inicial de la paciente

\begin{tabular}{|lcc|}
\hline Examen & Paciente & VN \\
\hline Creatinina $(\mathrm{mg} / \mathrm{dl})$ & 1,45 & $0,5-0,9$ \\
\hline Hematocrito $(\%)$ & 52,2 & $36-46$ \\
\hline Hemoglobina $(\mathrm{g} / \mathrm{dl})$ & 17,8 & $12-16$ \\
\hline VHS $(\mathrm{mm} / \mathrm{h})$ & 7 & $<30$ \\
\hline Glicemia $(\mathrm{mg} / \mathrm{dl})$ & 101 & $70-99$ \\
\hline Insulina $(\mathrm{mUl} / \mathrm{mL})$ & 15,2 & $<16$ \\
\hline HOMA & 3,79 & $<2,5$ \\
\hline Ácido úrico $(\mathrm{mg} / \mathrm{dl})$ & 6,9 & $2,3-6,6$ \\
\hline HbA1c $(\%)$ & 5,8 & $4-6$ \\
\hline Colesterol total $(\mathrm{mg} / \mathrm{dl})$ & 231 & $<200$ \\
\hline Colesterol LDL $(\mathrm{mg} / \mathrm{dl})$ & 137 & $<100$ \\
\hline Colesterol HDL $(\mathrm{mg} / \mathrm{dl})$ & 33 & $>40$ \\
\hline Triglicéridos $(\mathrm{mg} / \mathrm{dl})$ & 304 & $<150$ \\
\hline
\end{tabular}

Tabla 2. Estudio hormonal y marcadores tumorales iniciales de la paciente

\begin{tabular}{|lcc|}
\hline Examen & Paciente & $\begin{array}{c}\text { VN } \\
\text { (ajustado } \\
\text { a edad) }\end{array}$ \\
\hline TSH $(\mathrm{mUl} / \mathrm{ml})$ & 3,87 & $0,3-4,2$ \\
T4 Libre $(\mathrm{ng} / \mathrm{dl})$ & 1,25 & $0,93-1,7$ \\
\hline Prolactina $(\mathrm{ng} / \mathrm{ml})$ & 9,9 & $2,1-25$ \\
\hline Testosterona total $(\mathrm{ng} / \mathrm{dl})$ & 711 & $15-80$ \\
[nmol/l] & {$[24,6]$} & {$[0,52-2,77]$} \\
\hline SHBG $(\mathrm{nmol} / \mathrm{l})$ & 24,4 & $9,3-100$ \\
\hline IAL & 101 & $<4,5$ \\
\hline DHEA-S $(\mathrm{mg} / \mathrm{ml})$ & $<0,3$ & $0,35-4,3$ \\
\hline FSH $(\mathrm{mUl} / \mathrm{ml})$ & 16,9 & $23-116$ \\
\hline LH $(\mathrm{mUl} / \mathrm{ml})$ & 9,3 & $15,9-54$ \\
\hline Estradiol $(\mathrm{pg} / \mathrm{ml})$ & 48 & $<20$ \\
\hline Cortisol libre U $(\mu \mathrm{g} / 24$ hrs $)$ & 15,1 & $20-90$ \\
\hline hCG $(\mathrm{mUl} / \mathrm{ml})$ & $<5$ & $<5$ \\
\hline$\alpha F P(\mathrm{Ul} / \mathrm{ml})$ & 2,0 & $<10,9$ \\
\hline CEA $(\mathrm{ng} / \mathrm{ml})$ & 3,4 & $<5$ \\
\hline Ca-125 $(\mathrm{U} / \mathrm{ml})$ & 7,0 & $<40$ \\
\hline
\end{tabular}

$\mathrm{mm}$ (VN $15 \times 7 \mathrm{~mm}$ en postmenopausia) ${ }^{2}$. La ecotomografía ginecológica transvaginal confirmó estos hallazgos y detectó engrosamiento endometrial de $11 \mathrm{~mm}(\mathrm{VN}<5 \text { en postmenopausia })^{3}$. La biopsia endometrial dio salida a material escaso informado como mucus, células endocervicales dispersas y escasos polimorfonucleares.

Dado que no existía evidencia clínica, de laboratorio ni de imágenes compatibles con un origen suprarrenal del hiperandrogenismo, se realizó salpingo ooforectomía bilateral vía laparoscópica. El ovario derecho pesó 6,6 g y midió 28 x 25 mm, presentando una superficie irregular, amarillenta (Figura 3) y el ovario izquierdo pesó $3 \mathrm{~g}$ y midió 32 x $20 \mathrm{~mm}$ presentando superficie opaca, con la lesión quística ya descrita. La microscopia demostró un tumor de células de Leydig hiliar en el ovario derecho, que comprometía gran parte de esa gónada, formado por células poligonales con citoplasma eosinófilo claro, finamente granular, con núcleos de cromatina periférica y 1 ó 2 nucleolos, dispuestas en nidos irregulares y en cordones; no se identificaron cristales de Reinke (Figura 4). El ovario izquierdo presentaba hiperplasia estromal y una lesión quística.

La paciente evolucionó en forma favorable, siendo dada de alta al tercer día postoperatorio. Una semana post cirugía, la paciente relata decaimiento, disminución de la libido y aparición de síntomas vasomotores. Los exámenes un mes post cirugía demostraron normalización del hiperandrogenismo (testosterona total $19 \mathrm{ng} / \mathrm{dl}$, SHBG de 30,7 nmol/L e índice andrógenos libres de 2,0). Transcurridos 7 meses de la cirugía, la alopecía androgenética ha revertido en forma importante (Figura 2) y los síntomas vasomotores han cedido espontáneamente. La hemoglobina plasmática se ha normalizado $(14,2 \mathrm{gr} / \%)$ y el HOMA está en 3,9, colesterol total $235 \mathrm{mg} / \mathrm{dl}$, LDL $132 \mathrm{mg} / \mathrm{dl}$, HDL $39 \mathrm{mg} / \mathrm{dl}$, triglicéridos $321 \mathrm{mg} / \mathrm{dl}$. La testosterona total es $12 \mathrm{ng} / \mathrm{dl}$, estradiol $13 \mathrm{pg} / \mathrm{ml}, \mathrm{FSH}$ 60,4 $\mathrm{mUI} / \mathrm{ml}, \mathrm{LH} 43,8 \mathrm{mUI} / \mathrm{ml}$ y DHEAS en $0,3 \mathrm{ug} / \mathrm{ml}$.

\section{Discusión}

El presente caso correspondió a un tumor de células de Leydig hilar del ovario, causante de hiperandrogenismo y virilización en la postmenopausia. Esta es una patología extraordinariamente infrecuente, constituyendo sólo el $0,5 \%$ de todos 


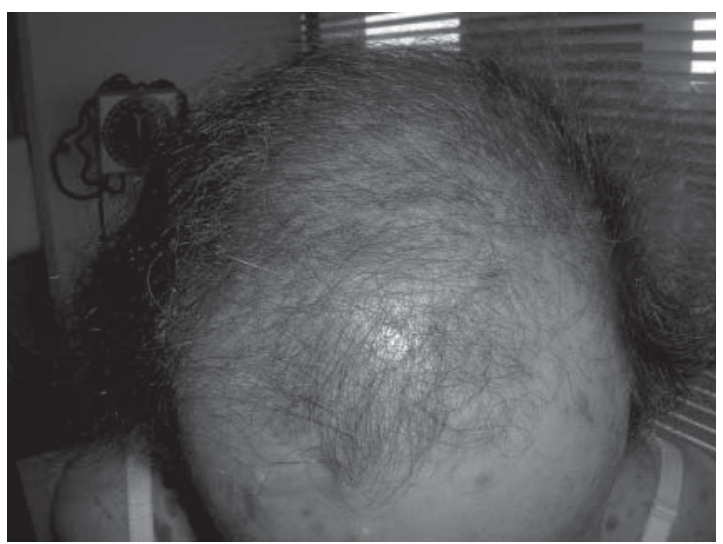

Figura 1. Alopecia previo a la cirugía.

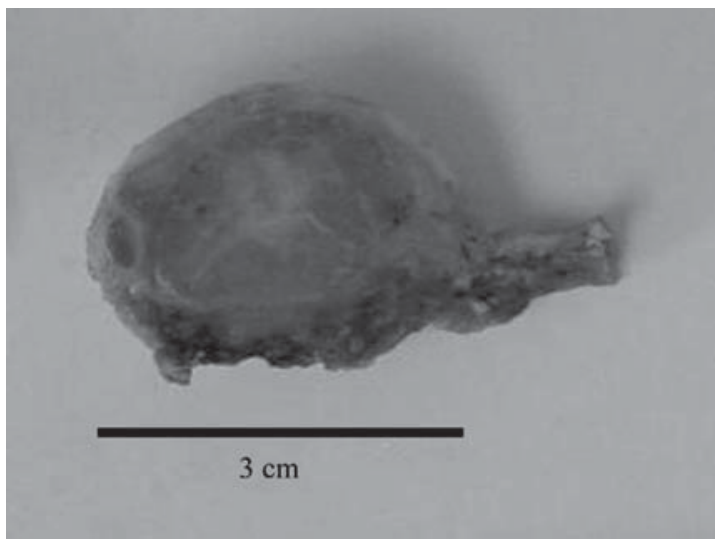

Figura 3. Macroscopia anexo derecho y tumor de células de Leydig hiliar.

los tumores ováricos y existiendo en la literatura sólo el reporte de casos esporádicos ${ }^{4-14}$. Una vez removido el tumor, los niveles de testosterona descendieron a valores propios de la postmenopausia y se inició un síndrome climatérico y un estado de trastorno del ánimo moderado, atribuible al brusco descenso hormonal. La paciente además presentaba una poliglobulia que revirtió, dislipidemia mixta y resistencia insulínica que se han mantenido estables transcurridos 7 meses desde la cirugía.

El hiperandrogenismo en la postmenopausia, y especialmente la virilización, es muy infrecuente y suele deberse a causas tumorales. Tanto la severidad del hiperandrogenismo, edad de la paciente, rapidez de instauración del cuadro y síntomas y

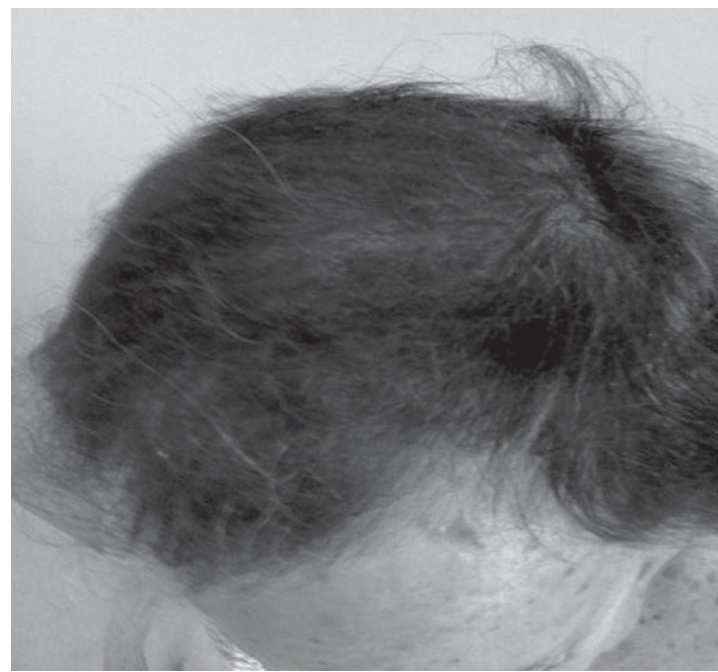

Figura 2. Alopecia posterior a la cirugía.

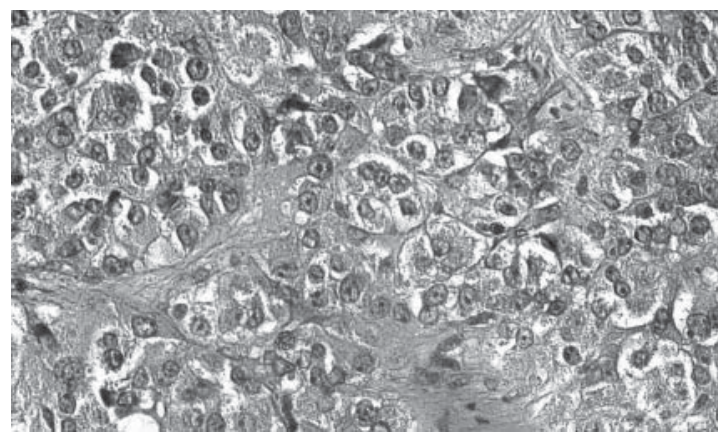

Figura 4. Histología de tumor de células de Leydig hiliar.

signos asociados son elementos importantes en el diagnóstico diferencial de este trastorno.

En la Tabla 3 se presentan las causas de hiperandrogenismo en la postmenopausia ${ }^{15}$. Eliminadas las causas iatrogénicas, las fuentes de origen probables son suprarrenales u ováricas, tanto benignas como malignas. Mucho menos posible es una causa ectópica, la que se ha descrito a nivel de músculo iliopsoas o intestinal ${ }^{4,13}$, y que suelen ser rápidamente detectada en la RM.

Hasta $30 \%$ de los tumores adrenales funcionantes producen virilización ${ }^{4}$, los que suelen secretar grandes cantidades de DHEA, DHEA-S y androstenediona, lo que no sucedía en nuestra paciente. La normalidad de cortisol libre urinario, hacía poco probable el origen suprarrenal, dado 
Tabla 3. Causas de hiperandrogenismo en la postmenopausia

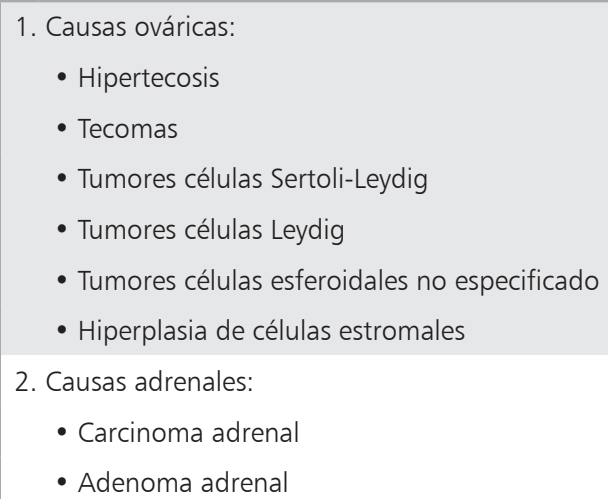

3. Fármacos: testosterona, glucocorticoides, danazol 4. Causas tumorales ectópicas

que los tumores adrenales virilizantes suelen tener secreción hormonal mixta ${ }^{1,16}$. Además, la inmensa mayoría de los tumores virilizantes corresponden a carcinomas suprarrenales y raramente a adenomas. En cualquiera de estas circunstancias, la RM de suprarrenales habría detectado la lesión suprarrenal, dado que los carcinomas, suelen ser mayores de $4 \mathrm{~cm}$ de diámetro ${ }^{17}$.

La otra fuente posible del hiperandrogenismo es el tejido ovárico. La mayoría de estos casos son benignos, y en esta paciente, su buen estado general y la evolución de alrededor de 2 años de su cuadro apuntaban en ese sentido. De las posibles etiologías ováricas descritas en la Tabla 1, la hipertecosis parece poco probable debido a que generalmente se asocia a una hiperinsulinemia grave $^{5}$. El presente caso orientaba fuertemente a una causa ovárica tumoral, situación que ocurre en menos de $5 \%$ de los tumores ováricos ${ }^{6}$. Descartadas otras etiologías descritas en la Tabla 1, especialmente un tumor de células de Sertoli Leydig dado que se presentan en la premenopausia en más de $90 \%$, suele alcanzar grandes tamaños con manifestaciones locales ${ }^{7-9,18}$, e incluso masa palpable, el cuadro era compatible con un tumor de Leydig hiliar. Más de 95\% son unilaterales y pueden ser muy pequeños e indetectables en la imagenología. Su comportamiento es benigno y el $75 \%$ se asocia a virilización. Si bien son característicos, los cristales de Reinke sólo se encuentran en poco más de la mitad de los $\operatorname{casos}^{9-11,19}$. Su alta tasa de secreción de andrógenos, se puede asociar a hiperestrogenismo mantenido originado en la conversión periférica de andrógenos a estrógenos, como sucedía en el presente caso, lo que implica mayor riesgo de hiperplasia endometrial e incluso cáncer endometrial.

En este caso había suficiente evidencia para descartar las causas suprarrenales y ectópicas. Por lo anterior se realizó ooforectomía bilateral. En los casos reportados, la decisión de ooforectomía también se debió a un diagnóstico de descarte ${ }^{1,5,11,12}$. Esta conducta tiene sustento en el conocimiento de la fisiología de la secreción androgénica. El principal andrógeno circulante en la mujer es la testosterona, que es secretada en $30 \%$ en forma directa por el ovario; el 70\% restante deriva de la conversión periférica de precursores ováricos y adrenales, siendo el más importante la androstenediona, secretada en cantidades equivalentes por ambas fuentes ${ }^{1}$.

Respecto a la presencia de un quiste simple de ovario de $20 \mathrm{~mm}$, si bien es infrecuente, constituyen $25 \%$ de los tumores ováricos en la postmenopausia ${ }^{20}$. De hecho, en nuestro caso, el tumor de la paciente se encontraba en el ovario contralateral al del quiste, el que tenía una imagen radiológica y ecográfica normal. Una forma más directa para hacer el diagnóstico diferencial y localizar la hipersecreción androgénica habría sido la cateterización simultánea de las venas ováricas y suprarrenales. No obstante, es invasivo, poco disponible y con morbilidad cercana al 5\% (rotura o trombosis de las venas cateterizadas). Por lo mismo, este es un procedimiento de excepción que no se justificaba en nuestro caso por lo evidente del origen del trastorno ${ }^{1,9,10,14}$.

Es interesante hacer notar que después de la ooforectomía, apareció un síndrome climatérico florido, decaimiento físico y disminución de su impulsividad y libido exacerbada, todo compatible con la desaparición del hiperandrogenismo. La alopecía revirtió y despareció la piel oleosa e hirsutismo facial.

El estradiol basalmente elevado $(48 \mathrm{pg} / \mathrm{ml})$ atribuible a aromatización periférica a partir de testosterona, descendió a niveles propios de la postmenopausia y se asoció a un incremento de FSH acorde a su edad.

En conclusión, presentamos un caso extraordinariamente infrecuente de virilización en la 
postmenopausia, en el que un estudio hormonal e imagenológico de uso habitual permitieron orientar hacia una causa ovárica, por descarte de las demás etiologías probables. La ooforectomía bilateral fue curativa.

\section{Referencias}

1. Bancos I, Prawius H. Leydig cell tumor of the ovary postmenopausal woman presenting with virilization. The Endocrinologist 2008; 18: 146-9.

2. Herrmann UJ. Sonographic patterns of ovarian tumors. Clin Obstet Gynecol 1993; 36: 375-83.

3. Bradley LD, Falcone T, Magen AB. Radiographic techniques for the diagnosis of abnormal uterine bleeding. Obstet Gynecol Clin North Am 2000; 27: 245-76.

4. Mavroudis K, Aloumanis K, Papapetrou P, Voros D, Spanos I. Virilization caused by an ectopic adrenal tumor located behind the iliopsoas muscle. Fertil Steril; 2007; 87: 1468.e 13-16.

5. García E, García-Hierro V, De la Maza L, Álvarez P, Santos E, Pi J, et al. Hiperandrogenismo en una mujer posmenopáusica. Endocrinol Nutr 2008; 55(8): 376-8.

6. Faraj G, Di Gregorio S, Misiunas A, Faure A, Villabrile P, Stringa I, et al. Virilizing ovarian tumor of cell type not otherwise specified: a case report. Gynecol Endocrinol 1998; 12: 347-52.

7. Lantzsch T, Stoerer S, Lawrenz K, Buchmann J, Strauss HG, Koelbl H. Sertoli-Leydig cell tumor. Arch Gynecol Obstet 2001; 264: 206-8.

8. Caringella A, Loizzi V, Resta L, Ferreri R, Loverro G. A case of Sertoli-Leydig cell tumor in a postmenopausal woman. Int J Gynecol Cancer 2006; 16: 435-8.

9. Regnier C, Bennet A, Malet D, Guez T, Plantavid M, Rochaix $\mathrm{P}$, et al. Intraoperative testosterone assay for virilizing ovarian tumor topographic assessment: Report of a Leydig cell tumor of the ovary in a premenopausal woman with an adrenal incidentaloma. J Clin Endocrinol Metab 2002; 87: 3074-7.
10. Ozgun MT, Batukan C, Turkyilmaz C, Dolanbay M, Mavili E. Selective ovarian vein sampling can be crucial to localize a Leydig cell tumor: An inusual case in a postmenopausal woman. Maturitas 2008; 61: 278-80.

11. Sanz OA, Rubio P, Troyas R, Iriarte MJ, Zambrano JL. Bilateral Leydig cell tumor of the ovary: A rare cause of virilization in postmenopausal patient. Maturitas 2007; 57: 214-6.

12. Reyes R, Quesada M, García G, Rozas P, Ochoa A. Hilus cell tumor in the ovary. The Endocrinologist 2007; 17: 152-3.

13. Bachelot A, Meduri G, Baudin E, Kuttenn F, Touraine P. Hyperandrogenism in a postmenopausal woman presenting with a metastatic ileum endocrine tumor. Fertil Steril 2004; 81: 675-8.

14. Dickerson R, Putman M, Black M, Pinto K, Diamond $\mathrm{N}$, Marynick S, et al. Selective ovarian vein sampling to localize a Leydig cell tumor. Fertil Steril 2005; 84: 218. e19-22.

15. Pugeat M, Raverot G, Plotton I, Brac de la Perrière A, Mirakian P, Déchaud H, et al. Androgen-secreting adrenal and ovarian neoplasms. Androgen Excess Disorders in Women, second edition; 2007: 75-84.

16. Brown J, Fishman L. Biosynthesis and metabolism of steroid hormones by human adrenal carcinomas. Braz J Med Biol Res 2000; 33: 1235-44.

17. Allolio B, Fassnacht M. Adrenocortical carcinoma: clinical update. J Clin Endocrinol Metab 2006; 91: 2027-37.

18. Young RH, Scully RE. Ovarian Sertoli-Leydig cell tumors: a clinicopathological analysis of 207 cases. Am J Gynecol Obstet 1985; 9: 543.

19. Paraskevas M, Scully RE. Hilus cell tumor of the ovary. A clinicopathological analysis of 12 Reinke crystal-positive and nine crystal-negative cases. Int J Gynecol Pathol 1989; 8 (4): 299-310.

20. Cuello M, Pomés C, Brañes J, Barrena N, Mayerson D, Wild R. Tumor ovárico en la postmenopausia. Consideraciones sobre su manejo actual. Rev Chil Obstet Ginecol 2003; 68 (2): 97-111. 